\title{
Bases para un estudio pormenorizado de la presencia del español y el portugués y de su incidencia real en el mundo
}

\author{
Daniel Héctor Prado ${ }^{1}$ (D) @ \\ Lía Varela ${ }^{2}$ (D) @ \\ ${ }^{1}$ Consultor internacional; ${ }^{2}$ Universidad Nacional de Tres de Febrero (UNTREF), Argentina.
}

Resumen. En las últimas décadas han circulado diversas estimaciones acerca de la importancia mundial de las lenguas, apoyadas en criterios, metodologías y fuentes de datos también diversos y no siempre confiables o actualizados. Dado el interés por conocer el estado actual y las proyecciones posibles de las lenguas iberoamericanas, este artículo propone, en su primera parte, una revisión crítica de los principales estudios cuantitativos existentes sobre las lenguas en el mundo. Seguidamente, y como resultado de esta revisión, ofrece un esbozo de instrumento para un estudio de la presencia del español y el portugués en el mundo, que puede asimismo dar elementos para una mejor comprensión de dinámicas culturales, educativas y otras que tienen lugar dentro del espacio iberoamericano y que se expresan en la esfera lingüística. La última parte del artículo presenta algunos ejemplos de análisis que pueden realizarse en esta perspectiva dentro del campo de la educación a partir de los datos estadísticos que se propone recabar.

Palabras clave: difusión de lenguas; estudios cuantitativos; español; portugués; espacio iberoamericano.

\begin{abstract}
Bases para um estudo detalhado da presença do espanhol e português e de seu impacto real no mundo

Resumo. Nas últimas décadas, têm circulado diversas estimativas sobre a importância mundial das línguas, baseadas em critérios, metodologias e fontes de dados que também são diversas e nem sempre confiáveis ou atualizadas. Dado o interesse em conhecer o estado atual e as possíveis projeções das línguas ibero-americanas, este artigo propõe, em sua primeira parte, uma revisão crítica dos principais estudos quantitativos existentes sobre as línguas no mundo. Posteriormente, e como resultado desta revisão, o trabalho oferece um esboço de um instrumento para um estudo da presença do espanhol e do português no mundo, o que também pode fornecer elementos para uma melhor compreensão das dinâmicas culturais, educacionais e outras que ocorrem dentro do espaço ibero-americano e que são manifestadas na esfera linguística. A última parte do artigo apresenta alguns exemplos de análises que podem ser realizadas nesta perspectiva no campo da educação, com base nos dados estatísticos propostos
\end{abstract}

Palavras-chave: difusão de línguas; estudos quantitativos; espanhol; português; espaço iberoamericano

\begin{abstract}
Basis for a detailed study of the presence of Spanish and Portuguese and its real incidence in the world

Abstract. In recent decades, various estimates have circulated about the global importance of languages, supported by criteria, methodologies and data sources that are also diverse and not always reliable or updated. Given the interest to know the current state and possible projections of Ibero-American languages, this article proposes, in its first part, a critical review of the main existing quantitative studies on languages in the world. Then, as a result of this review, it offers an outline of an instrument for a study of the presence of Spanish and Portuguese in the world, which can also provide elements for a better understanding of cultural, educational and other dynamics that take place within the Ibero-American space and that are expressed in the linguistic sphere. The last part of the article presents some examples of analysis that can be carried out in this perspective within the field of education based on the statistical data that it is proposed to collect.
\end{abstract}

Keywords: language spread; quantitative research; Spanish; Portuguese; ibero-american space. 


\section{Introducción}

Suelen circular en las redes y medios de comunicación unas estadísticas -organizadas por lo general en forma de rankings- acerca de las lenguas del mundo: las lenguas habladas en la mayor cantidad de países, o de mayor número de hablantes nativos, más extendidas en superficie, más aprendidas como lengua extranjera, entre otras. Los criterios aplicados así como las finalidades en que se inscriben tales clasificaciones varían en cada circunstancia de su puesta en discurso público o cotidiano. Vale decir que, en la marea incesante de información que nos rodea, el tema inevitablemente concita interés. A los hablantes de una lengua de gran difusión, estas cifras les permiten imaginar su comunidad de pertenencia (Anderson, 1983) extendida a la escala planetaria. Decisores gubernamentales y de las grandes empresas (en particular las tecnológicas, de comunicaciones, de medios, editoriales, de entretenimiento), por su parte, las leen en claves (geo)políticas y económicas.

La información cuantitativa tiene, en efecto, el valor de insumo en el momento de elaboración y de evaluación de estrategias de negocios (editoriales, de comercio electrónico, de turismo y otros) o de políticas públicas, que pueden ser sectoriales (acciones de fortalecimiento de enseñanza de la lengua, de armonización de terminologías, de creación de herramientas lingüísticas, entre otras), nacionales, regionales o globales (es el caso de acciones destinadas a lenguas en peligro de extinción, por ejemploº $)$. Dada la importancia y las dimensiones que asumen las iniciativas de estas características, la calidad de la información de partida resulta un factor crucial.

Frente al "saber popular" sobre el que se basan muchas de las estimaciones, diversos especialistas e instituciones han desarrollado diferentes metodologías, algunas rigurosas, otras menos, y publicado análisis cuyos resultados a veces son utilizados por la prensa con poca cautela, creando mitos y tendencias que terminan siendo contraproducentes en ciertas circunstancias.

Los indicadores que se consideran en las diferentes perspectivas analíticas son diversos como lo es la terminología utilizada en la presentación de los resultados. Así, unos autores hablarán del peso de cada lengua, de su valor económico, de su presencia, de su posición relativa o su influencia, según los énfasis de los enfoques respectivos.

${ }^{1}$ Señalemos el Atlas interactivo de las lenguas del mundo en peligro, patrocinado por la Unesco (Moseley, 2010), herramienta de referencia para los que alertan sobre las lenguas en extinción. 
En momentos en que resulta cada vez más necesario comprender las grandes tendencias mundiales en materia geo-lingüística y cultural para una adecuada proyección del conjunto de países hispano y luso-hablantes, conviene disponer de algunas claves para la lectura crítica de la información cuantitativa que sustenta los diversos rankings circulantes. Este artículo pretende hacer aportes en este sentido. En la primera parte se pasa revista a algunos estudios relevantes dedicados a las lenguas en la esfera internacional y global, con sus características y terminología particulares. En este recorrido buscaremos identificar herramientas de análisis que sean pertinentes para determinar la situación y los desafíos que enfrentan el español y el portugués en el mundo y, particularmente, dentro del espacio iberoamericano. Este será el objeto de la segunda parte. Por último, daremos algunos ejemplos de aprovechamiento de los datos estadísticos en el análisis de algunas cuestiones que se revelan a través de datos lingüísticos (o viceversa, que dejan ver, como contracara, fenómenos que tienen que ver con las lenguas) en el campo de la educación superior iberoamericana.

\section{Los estudios acerca de la importancia de las lenguas en el mundo}

Ante la marcada tendencia a sobrevalorar alguna o unas pocas cifras que pretenden dar cuenta del prestigio internacional ${ }^{2}$ de una lengua, han surgido, desde hace ya varias décadas, diferentes enfoques en estudios cuantitativos dedicados a detectar tendencias de desarrollo de un grupo lingüístico-cultural. Algunos buscan determinar la posición de una lengua en comparación con otras, y para ello observan el comportamiento de una serie de parámetros. Es el caso, entre otros, de los estudios de (Calvet, 2010; Chan, 2016; Lodares, 2001; Mora-Figueroa y Williams, 1996; Weber, 1997), el Global language network GLN (Ronen, Gonçalves, Hu, Vespignani, Pinker, y Hidalgo, 2014). Otros persiguen fines más específicos, como el de asignar un valor económico a cada lengua, como (Albuquerque, y Esperança, 2010; García, Alonso, y Jiménez, 2012; Grin, 2015; Reto, Azzim, Machado, y Costa, 2012) entre otros, o estimar la presencia de las lenguas en entornos determinados, como por ejemplo, el ciberespacio (Pimienta, Prado, y Blanco, 2010). En el mismo sentido, diferentes entidades de tutela lingüística han provocado o promovido informes y estudios sobre la incidencia de las lenguas

2 Es notorio el atajo que convierte al español, en contadas y respetadas

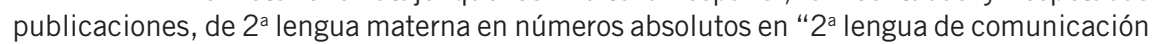
mundial", lo que es muy diferente y no verificado. 0 bien, de $3^{\text {a }}$ lengua en término de internautas en " $3^{a}$ lengua de Internet" lo que supone que los contenidos en español son equivalentes con el número de usuarios, lo que es totalmente falso. 
en el mundo. En particular, el British Council ${ }^{3}$ para el inglés, la Organisation internationale de la Francophonie $e^{4}$ para el francés, el Instituto Camões ${ }^{5}$ y la Comunidade dos paises de língua portuguesa ${ }^{6}$ para el portugués y el Instituto Cervantes $^{7}$ para el español, así como otros similares realizados por la Unión Latina $^{8}$, la Unesco ${ }^{9}$, la Unión europea ${ }^{10}$ y el Consejo de Europa ${ }^{11}$ en materia de multilingüismo. Aquellos que han buscado determinar la incidencia que puede tener una lengua a nivel internacional suelen tomar en cuenta factores demográficos, culturales, científico-tecnológicos, turísticos, económicos, geopolíticos, entre otros.

El Marqués de Tamarón (Mora-Figueroa y Williams, 1996) fue sin duda precursor cuando propuso el índice de importancia internacional de las lenguas en $1990^{12}$, que más tarde aplicó Jaime Otero y fue modificado por Moreno (2015). Este índice se basa en algunos parámetros como el número de hablantes nativos, el número de países en donde se habla, el índice de desarrollo humano (IDH), el volumen de exportaciones y de traducciones, así como su oficialidad en la ONU. Los seis indicadores son ponderados para obtener como resultado final un ranking en el cual la lengua española ocuparía el $3^{\circ}$ Iugar y el portugués, el $13^{\circ}$ lugar; modificando el sistema de ponderación, como él mismo propone, el español podría llegar al $2^{\circ}$ lugar, mientras que el portugués se mantendría en el mismo puesto. Georges Weber (1997) mide también el número de países donde es lengua oficial y el número de hablantes de lengua materna (indicado de ahora en más, en este texto, L1), y agrega los que la tienen como idioma vehicular (indicado de ahora en más, L2), el número de sectores principales en los cuales tiene un uso internacional, el poder económico, militar y financiero de los países hablantes de la lengua y, finalmente, el prestigio socio-literario que tiene la lengua a nivel internacional. De cada rubro se infiere un ranking específico (por ejemplo, lenguas más habladas o más enseñadas) y a cada rubro, Weber (1997) le asigna una ponderación específica ${ }^{13}$, para obtener un ranking de

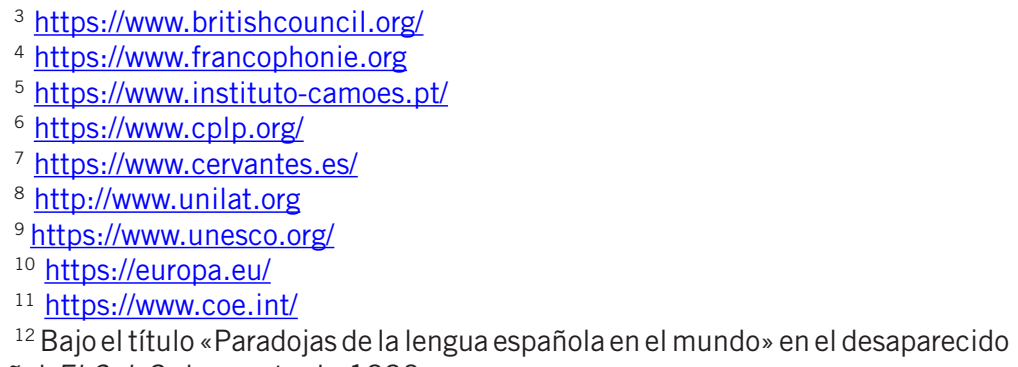
diario español El Sol, 3 de agosto de 1990.

${ }^{13}$ Hablantes maternos, 4 puntos; lengua vehicular, 6; poder económico, 8; importancia de la lengua por cantidad de áreas del conocimiento, 8; población y número de países en donde se habla, 7, prestigio socio-literario de la lengua, 4 puntos. Además, las lenguas oficiales en la ONU reciben 1 punto suplementario. 
las 10 lenguas más influyentes. Los trabajos posteriores han incorporado algunos de los parámetros de Tamarón y de Weber, mejorándolos, adaptándolos y agregando sectores de estudio. Por ejemplo, Lodares (2001) agrega la extensión geográfica de la lengua, su valor comercial y su estatuto oficial en organismos internacionales (incluido parcialmente en Weber).

Louis-Jean Calvet (Calvet, 2010), en un trabajo publicado inicialmente en el sitio Portalingua ${ }^{14}$ de la Unión Latina y accesible actualmente en un sitio del Ministerio francés de Cultura ${ }^{15}$, ha confeccionado ${ }^{16}$ lo que da en llamar un barómetro, en el cual, a los indicadores ya mencionados, agregan el número de artículos en Wikipedia, el número de premios literarios internacionales, el índice de fecundidad de su población, la entropía ${ }^{17}$, el índice de penetración de internet, la enseñanza de la lengua en el nivel universitario y el número de traducciones. Calvet (2010), sin embargo, no propone ninguna ponderación absoluta por parámetro, sino que deja al usuario la decisión de llegar a una clasificación específica según su interpretación de la valoración de cada indicador. Chan (2016), por su parte, habla de poder de la lengua en base a una clasificación en la que, además de incluir algunos de los parámetros anteriores, incluye la superficie de los países en los que se habla, el número de turistas que reciben, el producto nacional bruto (PNB) de los mismos, el número de sitios en ellos, la notoriedad de sus universidades, el número de hablantes por familia de lenguas, así como el número de largometrajes producidos en la lengua y su uso en la diplomacia. (Chan, 2016; Mora-Figueroa y Wiliams, 1996; Weber, 1997) definen una ponderación específica propia a cada indicador para llegar a su clasificación definitiva.

La empresa Bloomberg LP (2011) se enfoca en la construcción de un ranking de las "lenguas de negocios". Para ello, a algunos de los parámetros ya mencionados, se agregan el crecimiento económico de los últimos años, el grado de alfabetismo y de penetración de internet por habitante. También Bloomberg LP confecciona una ponderación propia.

${ }^{14}$ Consultable sólo en archivos: https://bit.ly/2Pdkyrw

${ }^{15}$ http://www.wikilf.culture.fr/barometre2017/index.php

${ }_{16}$ Con su hermano, Alain Calvet, matemático.

${ }^{17}$ En el Barómetro Calvet de las lenguas del mundo se da la siguiente definición del término: "La entropía es una función que permite cuantificar el desorden [...] la manera como [los] hablantes están repartidos en el área o las áreas en las que se habla". https:// bit.ly/2Ze3ZQ6 (p. 2) 
En la actual era digital, varias iniciativas en línea (GreffenstetteNioche ${ }^{18}$, InternetWorldStats ${ }^{19}$, Statista ${ }^{20}$, W3Techs ${ }^{21}$ y otros) proponen o han propuesto clasificaciones de las lenguas en Internet basadas en diferentes indicadores (como, por ejemplo, número de internautas, usuarios de Facebook o cantidad de contenido por lengua). Rojo y Sánchez (2010) realizaron para Telefónica un estudio sobre la situación del español en la red en el cual analizan una decena de parámetros (tecnológicos, de uso y sobre periodismo digital). Lo mismo se ha hecho en diferentes oportunidades para otras lenguas ibéricas como el catalán y el gallego. Pimienta y Prado (2010) han seguido la evolución de las lenguas romances en el ciberespacio desde 1998 hasta 2010 y luego, de todas las lenguas presentes en Internet desde 2012 hasta nuestros días. En esta última etapa hacen intervenir (para su último estudio editado parcialmente por la Francofonía ${ }^{22}$ ) hasta unos 350 indicadores diferentes, con el objetivo de evaluar el acceso, el uso y los contenidos de internet en las lenguas del mundo.

Hablar de índice de importancia internacional de las lenguas, de ranking de lenguas más influyentes, de peso de las lenguas, de poder de la lengua, de presencia de las lenguas, no significa otra cosa más que mostrar la incidencia que puede tener una o más lenguas con respecto a las otras.

Los estudios que intentan determinar el valor económico de una lengua se inscriben en cambio en una lógica no comparativa. En esta perspectiva se han propuesto sistemas de definición del valor que aporta el uso de una lengua a los países en los cuales ésta es oficial. Entre los pioneros de esta línea de trabajo se encuentra el suizo Grin (2015), quien estimaba en 17/18 mil millones de euros el ahorro que representaba para el Reino Unido la dominación de la lengua inglesa en Europa ${ }^{23}$. En este orden de ideas y a iniciativa de organismos y empresas españolas, García (2012) realizó una serie de estudios y coloquios que buscaron determinar el valor de la lengua en la economía del país. Es así como llega a la conclusión de que "la lengua castellana genera un $16 \%$ del PBI y del empleo en España". El Instituto Camões, por su parte, encomendó a Albuquerque y Esperança (2010) un estudio similar; éste llegó a la conclusión de que el portugués "representaba el $17 \%$ del PBI de Portugal." 24

\footnotetext{
18 https://arxiv.org/ftp/cs/papers/0006/0006032.pdf

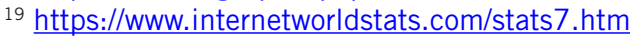

$20 \mathrm{https://bit.ly/31 \textrm {MMpQY }}$

${ }^{21}$ https://w3techs.com/technologies/overview/content language/all

22 Síntesis en http://observatoire.francophonie.org/2018/synthese.pdf

23 https://fr.m.wikipedia.org/wiki/Rapport Grin

24 https://bit.ly/30hwqtV
} 


\section{Para un estudio de la situación del español y portugués en el mundo}

Sin duda, la variedad de criterios utilizados -no siempre escrutados exhaustivamente-, así como la ausencia de fuentes siempre confiables y los intentos de obtener índices exactos a partir de la ponderación de indicadores -con todo el riesgo de subjetividad que eso presupone-, dejan una impresión de fragilidad al momento de determinar la influencia real de una lengua con respecto al resto.

Más lejana aún parece la posibilidad de determinar con alguna certeza el potencial de la relación entre las comunidades luso e hispanohablantes considerada desde la perspectiva de la cercanía entre ambas lenguas. De allí la propuesta que plantea esta contribución de una reflexión profunda sobre el cúmulo óptimo de indicadores, para el estudio de cada macro-sector considerado, necesarios para determinar la mayor o menor incidencia de una lengua (y, por ende, de los países o comunidades en los cuales es hablada), concretamente el español y el portugués, como también sobre aquellos indicadores que den cuenta del estado de las interacciones entre los espacios hispanohablante y lusófono.

Se trata de evaluar a partir de ellos la potencialidad de cada lengua en diferentes situaciones y dar lugar a una calificación global, que a sabiendas dará como resultado un rango y no una posición final. La idea no es proponer un índice absoluto de la capacidad de una lengua de incidir sobre las otras, sino valorar sector por sector esta incidencia, de manera de:

- evidenciar claramente los ámbitos en los cuales se perciben las mayores deficiencias, con el fin de provocar acciones de fortalecimiento, $y$

- apuntar a aquellos más dinámicos, de manera de identificar los argumentos que demuestren que la lengua en cuestión tiene un mayor prestigio, con fines de promoción de su aprendizaje, del turismo o del intercambio comercial, por ejemplo.

Con esta idea presentamos a continuación un relevamiento extenso de los indicadores que podrían revelar un posicionamiento global del español y del portugués (y de los países y comunidades que los hablan), dividido en macro-sectores. Estos indicadores deben ser medidos de manera idéntica para el conjunto de las lenguas tratadas, acudiendo a los más rigurosos relevamientos, encuestas, censos, estadísticas o estudios a los que sea posible acceder. 
Todos estos factores se pueden analizar en relación con el conjunto de las lenguas vivas a escala planetaria, para terminar seleccionando aquellas que manifiesten resultados comparables con el español y el portugués, así como aquellas lenguas que comparten territorialidad con éstas (por razones de extensión, no incluiremos aquí la totalidad de los indicadores ${ }^{25}$, sino simplemente los macro-sectores que los contienen).

a) La extensión geo y demo lingüística de las lenguas. Sin dudas, son los criterios más utilizados en toda evaluación del peso o prestigio de una lengua. Cuanto más hablada es una lengua, tanto en extensión geopolítica, como en número de hablantes L1 y L2, siempre se ha considerado que mayor será su influencia. Sin embargo, los factores deben ser múltiples y convergentes para que esa incidencia sea realmente efectiva. El hindi, por ejemplo, a pesar de ser una de las cinco lenguas más habladas en número, carece (entre otros aspectos) de la extensión geopolítica que conocen otras lenguas (español, portugués, ruso o árabe, por ejemplo). Así, se propone estudiar:

1. $N^{\circ}$ de hablantes $L 1$ en sus zonas de influencia.

2. Índice de fecundidad de su población

3. $N^{\circ}$ de hablantes $L 2$ en sus zonas de influencia.

4. Habitantes totales (incluyendo a los alófonos) en los países en los cuales son oficiales.

5. Extensión geográfica de dichos países.

6. Países y regiones en los cuales son lenguas oficiales (de jure o de facto)

7. Extensión de sus diásporas

b) La lengua en las relaciones internacionales. Otro gran revelador de la importancia de una lengua es su uso a nivel internacional. Las lenguas oficiales de las agencias de la ONU (o de organismos regionales, por caso), así como aquellas que son utilizadas para transacciones comerciales, han adquirido un prestigio internacional mayor. El portugués, por ejemplo, injustamente relegado en el momento de la creación de la ONU, encuentra progresivamente un lugar como lengua de trabajo, pero este lugar sigue aún siendo insuficiente si se toma en cuenta la dimensión demo-lingüística y geo-política de la lengua portuguesa. Se propone estudiar los siguientes indicadores:

1. Organismos en los cuales son oficiales o de trabajo

2. Uso en la diplomacia y la negociación internacional

3. Uso en las transacciones comerciales internacionales

${ }^{25}$ En el caso del ciberespacio, por ejemplo, se trata de más de 300 indicadores y/o micro-indicadores 
c) El peso económico del conjunto de sus países y de sus habitantes. El peso económico de las naciones o regiones en las cuales se habla una lengua incide en la necesidad de aprender dicha lengua. El mandarín desde hace siglos ha sido la lengua con mayor número de hablantes. Sin embargo, su enseñanza como lengua extranjera sólo se ha acelerado a causa del crecimiento económico de estas últimas décadas. Tampoco incide sólo la capacidad económica global (Producto interno bruto o PIB / Exportaciones / Turismo, entre otros), sino también la capacidad económica de cada uno de sus habitantes (Paridad de poder adquisitivo o PPA / Coeficiente de Gini). Estos son algunos de los indicadores por estudiar:

1. Producto Interno Bruto (PIB)

2. PIB per cápita

3. Paridad de poder adquisitivo (PPA)

4. Coeficiente de Gini

5. Volumen de exportaciones

6. Evaluación del peso turístico

7. Tendencias económicas en los últimos años

8. Volumen de comercio electrónico

d) La evaluación de parámetros sociales y humanos. Es importante evaluar las condiciones, tanto educativas como en materia de acceso a los medios y a la comunicación por parte de los hablantes de una lengua determinada, para observar si la misma puede desarrollarse en buenas condiciones. Estos son algunos de los indicadores por estudiar:

1. Índice de desarrollo humano (IDH)

2. Índice de educación

3. Acceso a la Universidad

4. Acceso a la telefonía

5. Acceso a radio y televisión

e) La presencia de las lenguas en el ciberespacio. La revolución digital ha impuesto nuevos modos de acceder a la información, de comunicarse, de aprender, de comprar, de entretenerse o de manifestarse, por ejemplo. Uno de los indicadores más elocuentes en cuanto al uso y prestigio de la lengua es hoy día, sin dudas, su presencia en Internet. Así, se estudiarán más de 300 micro-indicadores que permitan evaluar con precisión el lugar que ocupan las lenguas estudiadas, reunidos en macroindicadores tales como:

1. El acceso a la tecnología ( $N^{\circ}$ de internautas, tasa de penetración de internet, banda ancha, telefonía móvil, $\mathrm{N}^{\circ}$ de sitios/hab., entre otros) 
2. El uso de Internet (usuarios de portales, mensajerías, redes sociales, juegos, descargas, entre otros)

3. Los contenidos ( $\mathrm{N}^{\circ}$ de artículos en el mundo Wikimedia, $\mathrm{N}^{\circ}$ de libros en librerías virtuales, $\mathrm{N}^{\circ}$ de artículos científicos, entre otros)

4. Disponibilidad de redes sociales en la lengua (Facebook, LinkedIn, Twitter, Whatsapp, entre otros)

5. Disponibilidad de herramientas informáticas (plataformas, traducción automatizada, diccionarios y bases de datos, softwares lingüísticos, entre otros)

f) Lengua en la ciencia. A pesar de que los países de lengua española y portuguesa tienen una gran actividad de investigación científica, no podemos afirmar que sus lenguas gocen de un gran prestigio en el mundo científico en general, tanto en los índices como en los coloquios internacionales. A nivel universitario, incluso si los programas europeos han incentivado a muchos alófonos a estudiar en la Península Ibérica, las mismas universidades acuden cada vez más al recurso de enseñar en inglés, para incrementar ese flujo, en detrimento de sus lenguas nacionales. Estos son algunos de los indicadores por estudiar:

1. Posición de las lenguas en los grandes índices científicos

2. $\mathrm{N}^{\circ}$ de patentes internacionales

3. Lugar ocupado en la normativa internacional

4. Volumen de tesis producidas accesibles

g) La notoriedad de la producción cultural y científica. La notoriedad internacional de hombres y mujeres que participan en la creación tanto cultural como científica, aun cuando no traduzca necesariamente el volumen y la calidad de la producción de las naciones que representan, constituye un factor de atracción por el conocimiento de sus culturas y la apreciación de sus obras. Algunos indicadores:

1. Premios Nobel y grandes premios científicos

2. Premios literarios internacionales

3. Otros premios y galardones culturales (audiovisual, bellas artes, entre otros) internacionales

4. Notoriedad de universidades

5. Movilidad de estudiantes

h) Enseñanza de la lengua. El número de estudiantes de una lengua es, por un lado, un importante indicador de la vitalidad de esa lengua y, por otro lado, es probablemente el sector económico que más se ve beneficiado con el prestigio internacional de una lengua (Grin, 2015) Algunos indicadores:

1. $N^{0}$ de alfabetizados en la lengua

2. Enseñanza a nivel universitario

3. Enseñanza como lengua extranjera 
4. Número de certificados de nivel de conocimiento de la lengua

5. El grado de intercomprensión o proximidad con otras lenguas del mismo grupo lingüístico y comparación con otros grupos de lenguas.

6. Conocimiento y uso experto de las lenguas en el ámbito académico.

i) Las Industrias culturales. La música, el cine, las series o novelas, la literatura y otras obras creativas que participan en lo que se da en Ilamar las industrias culturales constituyen un atractivo fundamental en el deseo del conocimiento de la cultura de origen. Las novelas hispano-americanas o brasileñas, la música brasileña y el fado, el cine español y portugués, entre otras, han participado en buena medida en el auge de las lenguas de la región. Algunos indicadores:

1. Estadísticas sobre la incidencia de la música en el extranjero

2. $\mathrm{N}^{0}$ de largometrajes, documentales y series (novelas)

3. $\mathrm{N}^{0}$ de libros

4. $\mathrm{N}^{\circ}$ de traducciones hacia otras lenguas

En el siguiente punto mostraremos, a través de algunos ejemplos, el tipo de análisis a que pueden dar lugar datos estadísticos como los mencionados, en la perspectiva de conocer tendencias actuantes dentro del espacio iberoamericano y que se expresan también en la esfera de las lenguas. Mostraremos también de este modo la aplicación a escala regional de algunos de los indicadores mencionados. Por ejemplo:

- Tesis de posgrado sobre el español en países lusohablantes y viceversa

- Difusión de la/s otra/s lengua/s iberoamericanas en el espacio de la educación superior.

- Movilidad de estudiantes entre países del espacio iberoamericano

- Conocimiento y uso experto de las lenguas en el ámbito académico.

\section{Contactos e intercambios entre el español y el portugués en el espacio iberoamericano: ¿qué dicen los datos?}

En la búsqueda de claves para comprender las dinámicas que operan hoy en el espacio iberoamericano y vislumbrar proyecciones posibles, sería pertinente preguntarse, por ejemplo, por el grado de intensidad y amplitud que tienen los intercambios académicos, culturales, científicos, migratorios, comerciales hacia el interior del bloque, y cuáles son las circunstancias que favorecen o limitan el desarrollo de estos entramados. Dado que todos estos 
movimientos suponen intercambios lingüísticos y, por tanto, que al menos ciertos participantes conozcan la lengua del otro o incluso terminen por adoptarla, algunos de los indicadores que se tomen en cuenta para visibilizar la progresión de los intercambios en cada ámbito (número de estudiantes en movilidad, de publicaciones científicas conjuntas, flujos migratorios, turísticos u otras estadísticas referidas a consumos culturales, entre otros) podrían revelar también tendencias en cuanto a la extensión del conocimiento y uso de los idiomas del espacio iberoamericano en tales sectores. Los fríos números sin embargo pasarían por alto la infinidad de matices que componen cualquier imagen que se ofrezca de las prácticas en un idioma en contacto extendido con otro, con el que mantiene una relación horizontal y de proximidad genética, como es el caso del español y el portugués. Estudios de terreno, de corte etnográfico y cualitativo, sobre las prácticas lingüísticas en situaciones características tanto públicas como privadas darían sin duda un panorama diverso de usos y grados de conocimiento de la lengua del otro, en el que el ejercicio de la intercomprensión entre hablantes de una y otra lengua (por ejemplo, diálogos en los que cada participante habla su idioma y comprende el del otro) no serían en absoluto infrecuentes.

La difusión de las lenguas, revelador de la extensión o intensificación de los intercambios, lo es también del avance de un proceso de integración -política, social, económica-. Una manera clásica de representar la progresión de las lenguas en un territorio (sobre todo cuando se trata de lenguas internacionales como el español y el portugués) es, como se señalaba más atrás, la de observar el incremento en el número de personas que aprenden el idioma en cuestión en tanto lengua segunda o extranjera, o bien que obtienen certificaciones de nivel de conocimiento en los países considerados. Por ejemplo, de la información de esta naturaleza reunida a partir de diversas fuentes ${ }^{26}$, referida a la situación del portugués en Argentina, se pueden hacer observaciones como las siguientes: si bien a nivel nacional se observa un lento avance en el número de estudiantes de portugués en la educación formal, la situación es mucho más dinámica en algunos sistemas educativos provinciales.

26 Varela (2014), Nández y Varela (2015); información estadística referida a matrícula escolar y de los institutos de formación de profesores proporcionada por la Dirección Nacional de Investigación y Evaluación de la Calidad de la Educación (DINIECE) del Ministerio de Educación; información sobre matrícula universitaria provista por el Departamento de Información Universitaria, Secretaría de Políticas Universitarias, Ministerio de Educación; encuesta sobre usos y conocimientos lingüísticos entre estudiantes de la Universidad Nacional de Tres de Febrero (proyecto de investigación "Política lingüística e internacionalización de la Educación Superior, Varela dir. 2016-2020); datos sobre matrícula y número de cursos de portugués del Centro Universitario de Idiomas (CUI, http://www.cui.edu.ar); datos de matrícula del Instituto de Idiomas de Salta (http://www. edusalta.gov.ar/index.php/servicios/instituto-de-idiomas), entre otros. 
La oferta de formación de profesores se ha ampliado, si bien el número de egresados mantenía la tendencia decreciente que se verifica en la formación docente en general. La demanda de formación en portugués, en cambio, ha crecido significativamente en la educación superior (carreras de formación de profesores de otras disciplinas, donde es obligatorio el aprendizaje de una lengua extranjera; carreras de turismo y hotelería, de comercio internacional, de relaciones internacionales, escuelas de enfermería, policía y gendarmería, entre otras), así como en capacitación laboral en empresas y la administración pública. Por estas razones, y también debido a la frecuencia del aprendizaje informal en contextos familiares, de turismo y de movilidad por estudios, entre otros, el portugués se va instalando como segunda lengua extranjera más presente en los repertorios lingüísticos (lo que es particularmente válido entre las jóvenes generaciones) y más demandada en numerosos contextos institucionales, desplazando al francés de un lugar que éste había ocupado por décadas.

La proyección cartográfica de estos indicios de prácticas lingüísticas resultaría sumamente reveladora de procesos económicos, sociales, culturales y educativos en curso. Los mapas incluidos en Varela (2014), construidos a partir de datos de 2010 ya evidenciaban una clara tendencia en el avance de este idioma en Argentina. El crecimiento afectaba en primer lugar y más intensamente a las provincias limítrofes de la cuenca del Paraná (Misiones, Corrientes), luego el entorno de los ejes fluviales, aéreos y viales que conectan los principales nodos comerciales de Argentina, Bolivia, Brasil y Chile, más tarde el de los destinos más frecuentados por los turistas provenientes de los países vecinos. Zonas más distantes de estas corrientes de intercambio (en la Patagonia, por ejemplo), permanecían en cambio aún bastante ajenas a la difusión del portugués. La imagen que componen estos movimientos, similares a los que tienen lugar en otros países del Cono Sur, se deja leer fácilmente como una radiografía del proceso de integración social, cultural y económica en curso en la región.

En este orden de ideas, el número de tesis de posgrado centradas en el estudio de un aspecto específico de la/s lengua/s de otro/s país/es iberoamericano/s puede ser revelador del grado de interés, uso y conocimiento experto -en este caso- de tal idioma en determinado país. Las series anuales pueden mostrar, además, la evolución de este interés a lo largo del tiempo, y dar lugar a la formulación de hipótesis explicativas. Con esta intención realizamos una búsqueda de tesis de doctorado y disertaciones de maestría referidas a la lengua española en el Catálogo de la CAPES brasileña ${ }^{27}$. El

27 http://catalogodeteses.capes.gov.br/catalogo-teses/\#!/ 
análisis cuantitativo sobre las más de 4000 tesis y disertaciones que incluyen "língua espanhola" en el descriptor de su objeto, defendidas en el período 1987-2016²8, arroja la siguiente progresión:

Gráfico 1. Tesis y disertaciones en relación con la lengua española defendidas en Brasil, 1987-2016

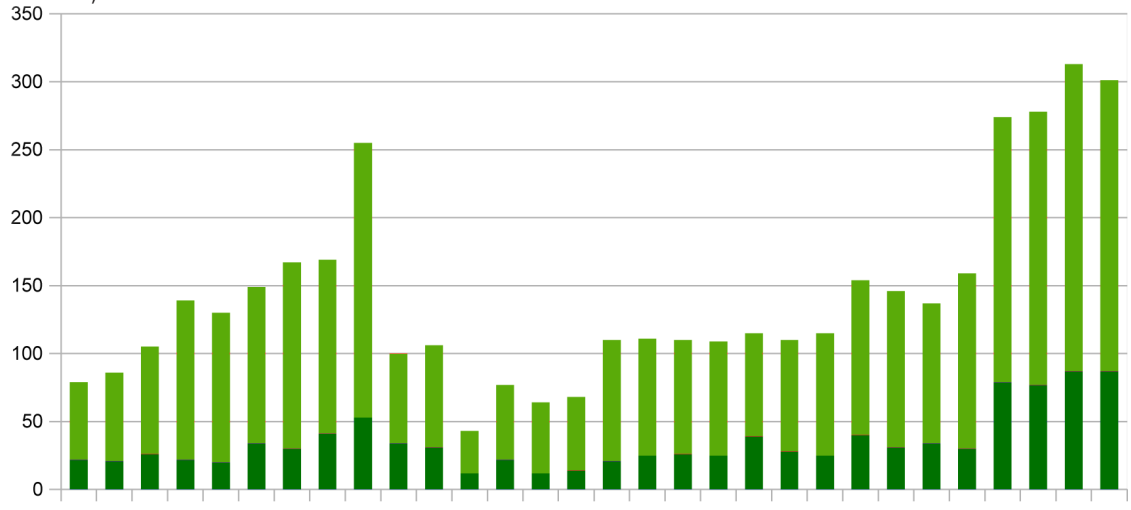

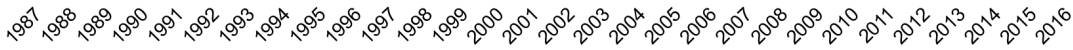

Tesis de doctorado Disertaciones de maestría

Fuente: Catálogo de Teses, CAPES, Brasil. Consultado en junio 2019.

La curva muestra un movimiento ascendente al inicio, con un pico excepcional en 1995 y una abrupta caída a partir del año siguiente, situación que no remonta hasta 2002. Este año la curva se ameseta y se mantiene modesta y constante hasta 2008. A partir de ese año se observan dos períodos diferenciados: un nuevo incremento que se estabiliza durante el período 2009-2012, luego un importante crecimiento, sostenido hasta 2016, año a partir del cual las cifras parecen indicar una nueva tendencia a la baja.

Las oscilaciones que detectamos en esta curva se pueden leer en clave histórica, y especialmente en relación con cambios en la política exterior referida a las relaciones del país con sus vecinos hispanohablantes. La firma del Acta de Integración Argentino-Brasileña en 1986 marca el inicio de un período de acercamiento del Brasil con respecto a los países vecinos que se formaliza en 1991 con la constitución del MERCOSUR. El entusiasmo por las posibilidades de estrechamiento de vínculos en la región parece haber alentado el interés por estudios especializados en el área de español. El cambio de orientación política ocurrido durante el gobierno de F.H. Cardoso (1994-2002) se traduce por una pérdida de este interés, que vuelve a manifestarse con la llegada de Lula da Silva a la presidencia (2003-2010), y en de CAPES.

${ }^{28} \mathrm{El}$ período citado comprende la totalidad de los datos contenidos en la base 
particular a partir de 2008, año de creación de la UNASUR. La aprobación de la Ley de enseñanza obligatoria de español en $2005^{29}$, como los desafíos de su implementación, pueden haber tenido un impacto mediato en el crecimiento del interés académico por el español. Bajo la presidencia de Dilma Rousseff (2011-2016), y junto con la prioridad que adquiere el MERCOSUR en la agenda de política internacional, el tema experimenta un auge notable.

Una elaboración a partir de las estadísticas referidas a la movilidad entrante y saliente de estudiantes universitarios contenidas en las bases de datos de UNESCO será el próximo ejemplo de aprovechamiento de datos existentes para el estudio de dinámicas sociohistóricas, culturales, educativas o económicas, con impacto en el terreno de las lenguas, dentro del espacio iberoamericano. A pesar de las limitaciones que implica el hecho de que no todos los países hacen público el número y procedencia de estudiantes extranjeros en sus aulas ${ }^{30}$, la información reunida en la tabla 1 a continuación revela tendencias consistentes.

Tabla 1. Movilidad saliente de estudiantes entre países iberoamericanos

\begin{tabular}{|c|c|c|c|c|c|c|c|c|c|c|c|c|c|c|c|}
\hline \multirow[b]{2}{*}{ 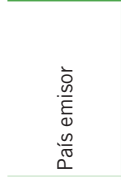 } & \multirow[b]{2}{*}{ 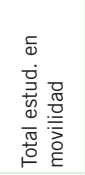 } & \multicolumn{12}{|c|}{ Países receptores } & \multirow[b]{2}{*}{ 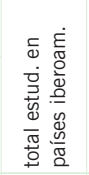 } & \multirow{2}{*}{ 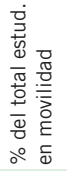 } \\
\hline & & 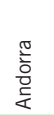 & 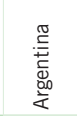 & $\begin{array}{l}\overline{\bar{n}} \\
\stackrel{\bar{\nu}}{\bar{\infty}}\end{array}$ & $\begin{array}{l}\frac{\pi}{0} \\
\frac{0}{E} \\
\frac{0}{0}\end{array}$ & $\begin{array}{l}\tilde{J} \\
\frac{0}{\pi} \\
\widetilde{J} \\
\tilde{J} \\
0\end{array}$ & $\frac{\omega}{\frac{\pi}{U}}$ & 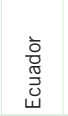 & 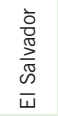 & 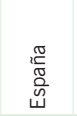 & $\begin{array}{l}\mathscr{0} \\
\stackrel{0}{5} \\
\overline{0} \\
\text { 오 } \\
\text { 온 }\end{array}$ & 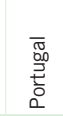 & 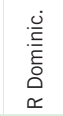 & & \\
\hline Andorra & 1410 & & 6 & 7 & & & & 7 & & 989 & & 13 & & & \\
\hline Argentina & 8371 & & & 1060 & 116 & 25 & 125 & 103 & 5 & 1050 & 9 & 37 & 34 & 2564 & 30,62 \\
\hline Bolivia & 19107 & 5 & 9966 & 944 & 45 & 15 & 304 & 74 & & 690 & 47 & 8 & 6 & 12104 & 63,34 \\
\hline Brasil & 52515 & & 9219 & & 128 & 18 & 178 & 55 & 8 & 1281 & 6 & 6372 & 21 & 17286 & 32,91 \\
\hline Colombia & 36626 & & 7225 & 1681 & & 280 & 994 & 2080 & 32 & 4713 & 67 & 106 & 200 & 17378 & 47,44 \\
\hline Costa Rica & 2843 & & 203 & 60 & 59 & & 35 & 18 & 11 & 296 & 26 & 13 & & 721 & 25,36 \\
\hline Cuba & 2288 & & 163 & 280 & 65 & 30 & 77 & 174 & & 362 & 5 & 31 & 161 & 1348 & 58,91 \\
\hline Chile & 14122 & & 5236 & 530 & 126 & 15 & & 142 & 6 & 1316 & 6 & 26 & 13 & 7416 & 52,51 \\
\hline Ecuador & 19324 & & 2278 & 351 & 531 & 146 & 421 & & 9 & 5318 & 420 & 58 & 32 & 9564 & 49,49 \\
\hline El Salvador & 4416 & & 216 & 53 & 37 & 133 & 37 & 11 & & 340 & 183 & 8 & 18 & 1036 & 23,46 \\
\hline España & 37723 & 138 & 791 & 275 & 97 & 30 & 68 & 104 & 6 & & & 1008 & 129 & 2646 & 7,01 \\
\hline Guatemala & 3495 & & 137 & 31 & 18 & 80 & 70 & 6 & 143 & 244 & 247 & 6 & 17 & 999 & 28,58 \\
\hline Honduras & 4593 & & 119 & 104 & 46 & 95 & 26 & 18 & 149 & 326 & & & 20 & 903 & 19,66 \\
\hline México & 33854 & & 523 & 218 & 530 & 68 & 103 & 96 & 32 & 2447 & 39 & 55 & 46 & 4157 & 12,27 \\
\hline Nicaragua & 3555 & & 35 & 35 & 22 & 1397 & 14 & 8 & 79 & 184 & 67 & & 16 & 1857 & 52,23 \\
\hline Panamá & 3429 & & 142 & 31 & 123 & 37 & 74 & 8 & 34 & 253 & 76 & 6 & 23 & 807 & 23,53 \\
\hline Paraguay & 12435 & & 9361 & 1160 & 16 & 7 & 34 & 5 & & 288 & & 9 & 6 & 10886 & 87,54 \\
\hline Perú & 30591 & & 14772 & 1437 & 322 & 58 & 1214 & 265 & 12 & 2105 & 52 & 41 & 58 & 20336 & 66,48 \\
\hline Portugal & 12713 & & 25 & 727 & 5 & & & & & 1586 & & & 7 & 2350 & 18,48 \\
\hline R. Dominic. & 4048 & & 202 & 50 & 49 & 35 & 34 & 11 & 8 & 1099 & 34 & 5 & & 1527 & 37,72 \\
\hline Uruguay & 4630 & & 2648 & 584 & 13 & 11 & 30 & 13 & 5 & 231 & & 8 & & 3543 & 76,52 \\
\hline Venezuela & 18107 & & 1633 & 299 & 1462 & 118 & 299 & 423 & 11 & 1369 & 6 & 137 & 334 & 6091 & 33,64 \\
\hline Total & 330195 & 143 & 64900 & 9917 & 3810 & 2598 & 4137 & 3621 & 550 & 26487 & 1290 & 7947 & 1141 & 127563 & 38,63 \\
\hline
\end{tabular}

29 Ley $n^{0} 11.161$ del 5/8/2005, revocada por ley n 13.415 de 2017.

${ }^{30}$ Los países ausentes en el eje horizontal son aquellos que no comunican datos de movilidad entrante o bien que no reciben estudiantes extranjeros. 
Se observará, por ejemplo, que dos países reciben el mayor flujo de estudiantes provenientes de todos los países iberoamericanos: Argentina, con cerca de 65.000 estudiantes iberoamericanos y España, con menos de la mitad de esta cifra (26.500). Entre ambos países reúnen casi un tercio de los estudiantes iberoamericanos que migran para continuar sus estudios.

La comunidad lingüística resulta evidentemente un factor de peso a la hora de elegir destino para una movilidad académica. De los 127.563 jóvenes que continúan estudios en otro país iberoamericano, el $80,8 \%$ se instala en un país de su mismo idioma. Entre los que cambian de idioma, son más numerosos los estudiantes lusófonos que se instalan en país hispanohablante (12.537) que los hablantes de español en Brasil o Portugal (10.765). Esta cifra representa sin embargo el $60,26 \%$ de los estudiantes iberoamericanos que reciben ambos países.

Las cifras de nuestra tabla también revelan dinámicas diferenciadas entre los diversos países en cuanto a elección de destino. Se observan allí, por ejemplo, países que reciben más estudiantes iberoamericanos que los nacionales que migran a tales países. El caso extremo es Argentina, con 64.900 entrantes frente a 2564 salientes, que se destinan casi en mitades iguales a universidades españolas y brasileñas. Los países que tienen la mayor proporción de estudiantes movilizados hacia países iberoamericanos (Paraguay, el 87,54\%; Uruguay, el 76,52\%; Perú, el 66,48\%; Bolivia, el 63,34\%), en tanto, no informan del número de estudiantes extranjeros en sus aulas. Los flujos de entrada y salida en estos casos no parecen ser equilibrados. España, por su parte, es el país que envía menos estudiantes a otros países iberoamericanos (2646, esto es, el $7 \%$ de sus estudiantes en movilidad). Estudiantes de ciertos países (Paraguay, Uruguay, Bolivia; Nicaragua) manifiestan una tendencia a la migración en el entorno próximo, hacia países que parecen funcionar como polos regionales (Argentina y Costa Rica, por ejemplo). En otros casos, donde se observa también un fuerte tropismo iberoamericano, se orientan hacia destinos más diversificados (por ejemplo, los estudiantes de Perú, Cuba o Colombia). La región andina (Chile, Perú, Ecuador, Colombia) revela un entramado intenso de movilidades recíprocas. Finalmente, España, Argentina y Brasil aparecen como los países receptores de contingentes iberoamericanos de procedencias más diversas.

\section{Conclusiones}

La presentación de un panorama de los estudios cuantitativos sobre la incidencia de las lenguas en el mundo que ocupó la primera parte de este artículo tenía el propósito de recordar la necesidad de contar no solamente con información confiable para cualquier evaluación sobre el estado y las perspectivas de una lengua, sino también con un número óptimo y elocuente de 
indicadores que sustenten los análisis. Mediante una breve reseña de casos -el avance desigual del portugués en Argentina, las fluctuaciones en el interés por la lengua española como objeto de estudio experto en el campo universitario brasileño y el factor lingüístico en la movilidad de estudiantes en el espacio iberoamericano-, creemos haber podido ilustrar luego el potencial de este tipo de estudios para visibilizar dinámicas que están operando actualmente en este espacio y en el mundo en relación a las lenguas del bloque. Análisis interdisciplinarios de la información estadística que proponemos recolectar, idealmente en diálogo con abordajes cualitativos, pueden colaboran así en la detección y la interpretación de fenómenos que demandan intervención. Para una formulación pertinente y adecuada a los fines establecidos, las políticas públicas que atiendan las diversas problemáticas vinculadas con las lenguas en la región deben partir del conocimiento de la situación real de las mismas. Este desafío se enfrenta hoy a la multiplicidad de fuentes de información, la caducidad de algunas de ellas, la diversidad de grados de comparabilidad y accesibilidad de los datos y la insuficiencia de parámetros utilizados hasta el presente.

\section{Referencias bibliográficas}

Albuquerque A., y Esperança, J. P. (2010). El valor económico del portugués: lengua de conocimiento con influencia global (sitio). Real Instituto Elcano. Recuperado de https://bit.ly/2ZaVLbr

Anderson, B. (1983). Imagined Communities. Reflections on the Origin and Spread of Nationalism, Verso, Londres-Nueva York.

Bloomberg LP. (2011). The languages of business (sitio). Bloomberg LP. Recuperado de https:// bit.ly/33Icrqn

Calvet, L. J. (2010). Poids des langues et « prospective »: essai d'application au français, à l'espagnol et au portugais. Synergies Brésil, 1, 41-58

Chan K. L. (2016). Power language index. Blog. Recuperado de https://bit.ly/30gZgdT

García Delgado J. L., Alonso J. A., y Jiménez, J. C. (2012). El valor económico del español. Madrid: Telefónica.

Grin, F. (2015). La valeur des langues dans l'activité professionnelle (Sitio) Fondation pour les études et recherches sur le développement international.

Lodares, J. R. (2001). Gente de Cervantes: Historia humana del idioma español. Madrid: Taurus Ediciones.

Mora-Figueroa y Williams, S. / Marqués De Tamarón (1996). El peso de la lengua española en el mundo. Valladolid: Ediciones Universidad De Valladolid 
Moreno, F. (2015). La importancia internacional de las lenguas. Informes del observatorio. Observatorio de la lengua española y las culturas hispánicas en los Estados Unidos. Recuperado de https://bit.ly/2s6R8Nf

Moseley, C (ed.) (2010). Atlas de las lenguas del mundo en peligro, 3ra edición. París, Ediciones UNESCO. Disponible en https://bit.ly/1RBHesp

Nández, J., y Varela, L. (eds.) (2015). Español y portugués, vectores de integración regional. Aportes para una política de formación docente. Buenos Aires: Teseo/PASEM.

Pimienta, D., Prado, D., y Blanco, A. (2010). Douze années de mesure de la diversité linguistique sur I'Internet: bilan et perspectives. París: UNESCO.

Reto, L., Azzim Gulamhussen, M., Machado, F., Costa, A.. (2012). Potencial Económico da Língua Portuguesa. Texto Editores, Lda.

Rojo, G., y Sánchez, M. (2010). El español en la red. Barcelona: Ariel y Fundación Telefónica.

Ronen S., Gonçalves B., Hu K. Z., Vespignani A., Pinker S., y Hidalgo, C. A. (2014). Links that speak: The global language network and its association with global fame. Proceedings of the National Academy of Sciences, 111(52), E5616-E5622. Disponible en https://bit.ly/2KJiUK9

Varela, L. (2014). Para un plan de implementación de la ley de portugués. E. Arnoux y S. Nothstein (eds.). Temas de Glotopolítica. (121-150). Buenos Aires: Biblos.

Weber, G. (1997). The Word's 10 Most Influential Languages. Language today, 2. 